Trauma Berufskrankh 2006 • 8[Suppl 3]:S317-S324 DOI 10.1007/s10039-005-1058-y

Online publiziert: 21. Dezember 2005

๑) Springer Medizin Verlag 2005

\author{
W. Strecker ${ }^{1} \cdot$ P. Keppler ${ }^{2}$ \\ ${ }^{1}$ Klinik für Orthopädische Chirurgie und Unfallchirurgie, Klinikum Bamberg \\ ${ }^{2}$ Klinik für Unfallchirurgie, Hand-, Plastische und Wiederherstellungschirurgie, \\ Universitätsklinik, Ulm
}

\title{
Therapie posttraumatischer Deformitäten
}

Weichteile, des Periosts und des Medullarsystems durchtrennt.

- Kompaktotomie

Die dünne metaphysäre Kortikalis und die spongiösen Randbereiche werden unter Anwendung minimalinvasiver Techniken mit Schonung der umgebenden Weichteile, des Periosts und des metaphysären Querschnitts durchtrennt.

\section{- Osteoklasie}

Dieser Begriff steht für einen kontrollierten Knochenbruch unter Schonung der umgebenden Weichteile, des Periosts und des Medullarsystems.

\section{Definitionen}

Die verschiedenen Arten der Knochendurchtrennung wurden von Baumgart et al. [1] unter Berücksichtigung der anatomischen Lokalisation, der periostalen und medullären Durchblutung sowie der Operationstechnik folgendermaßen definiert:

\section{- Osteotomie}

Es handelt sich um eine vollständige

Knochendurchtrennung über den gesamten Querschnitt ohne Unterscheidung zwischen Dia- und Metaphyse. Das Medullarsystem wird durchtrennt, die umgebenden Weichteile und das Periost werden entsprechend den allgemeinen Operationstechniken geschont.

\section{- Kortikotomie}

Die diaphysäre Kortikalis wird unter Anwendung minimalinvasiver Techniken mit Schonung der umgebenden

\section{Wahl des Durchtrennungs- verfahrens}

Die jeweiligen Korrekturziele und die gewählten Verfahren der Osteosynthese entscheiden letztlich über die Form der Knochendurchtrennung.

Einzeitige interne Osteosynthesen verlangen meist eine zimmermannsmäßige Umsetzung der Operationsplanung und damit exakt durchgeführte plane Schnitte. Die osteotomierten Flächen sollen passgenau aneinander liegen und durch die Osteosynthese flächig komprimiert werden. Diese angestrebte Präzision und Passgenauigkeit gehen jedoch zu Lasten der lokalen Biologie, kompromittiert durch die erhöhte Invasivität des operativen Zugangs, der Osteotomie mit der oszillierenden Säge und der nachfolgenden Osteosynthese.

Externe Korrekturtechniken sind bezüglich Knochendurchtrennung und Os- teosynthese im Allgemeinen weniger invasiv. Ihre Nachteile liegen in geringerem $\mathrm{Pa}$ tientenkomfort bei oft sehr langen Tragezeiten der externen Fixationssysteme, hohen Raten an Pininfekten und häufigen Weichteilirritationen mit konsekutiven Vernarbungen und Bewegungseinschränkungen.

Die unten dargestellten Osteotomieformen betreffen vorrangig interne Korrekturverfahren sowie die jeweiligen Indikationen. Die später detailliert beschriebenen Techniken der Knochendurchtrennung werden bezüglich ihrer Vor- und Nachteile für interne und externe Korrekturen gewertet.

\section{Osteotomieformen und Indikationen}

Quere Osteotomien. Sie bieten sich für Torsionskorrekturen an. Die Schnittfläche sollte rechtwinklig zur anatomischen Achse stehen und möglichst glatt und eben sein. Zur Knochendurchtrennung eignen sich oszillierende oder Gigli-Säge (• Abb. 1f, g). Bei geplanter Kallusdistraktion wird die quere Osteotomie aus biologischen Gründen bevorzugt mit Bohrer und/oder Meißel ausgeführt. Ausnahmsweise können derartige „approximative“ Osteotomien auch für die einzeitige Korrektur diaphysärer Deformitäten in der Frontal- oder Sagittalebene akzeptiert werden. Voraussetzung sind eine zuverlässige Ausgradung und Knochenheilung mittels gebohrter Marknagelung. 

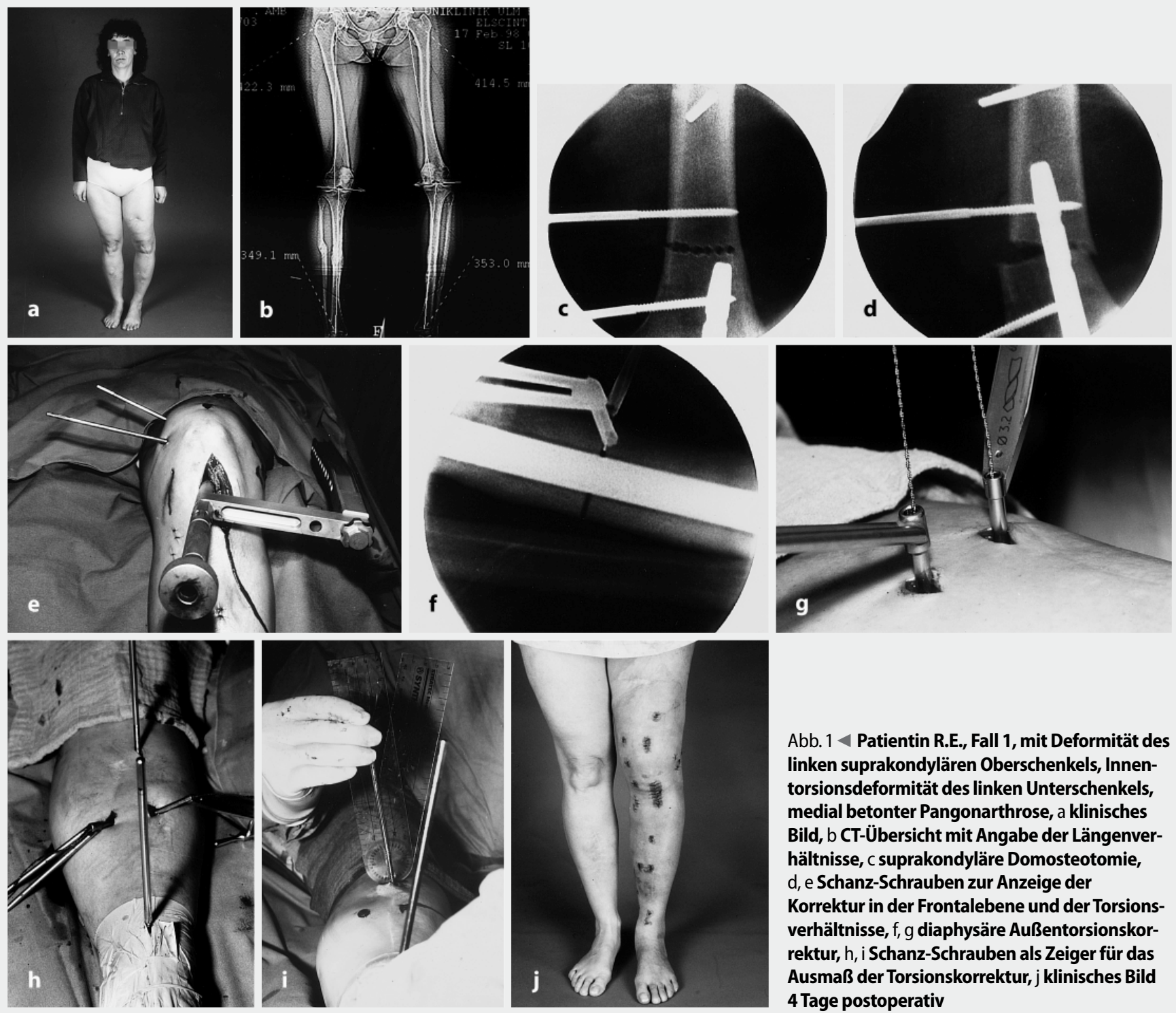

Abb. $1<$ Patientin R.E., Fall 1, mit Deformität des linken suprakondylären Oberschenkels, Innentorsionsdeformität des linken Unterschenkels, medial betonter Pangonarthrose, a klinisches Bild, b CT-Übersicht mit Angabe der Längenverhältnisse, c suprakondyläre Domosteotomie, $d$, e Schanz-Schrauben zur Anzeige der Korrektur in der Frontalebene und der Torsionsverhältnisse, $f, g$ diaphysäre Außentorsionskorrektur, $\mathrm{h}$, i Schanz-Schrauben als Zeiger für das Ausmaß der Torsionskorrektur, j klinisches Bild 4 Tage postoperativ

\section{Additive oder subtraktive Keilosteotomi-} en. Sie eignen sich für frontale und sagittale Achskorrekturen, Translationen und Torsionskorrekturen. Bevorzugtes Werkzeug für die erforderlichen glatten Schnitte ist die oszillierende Säge, wobei das Sägeblatt durch Anlegen an einen zuvor eingebrachten Kirschner-Draht dirigiert wird. Bei eindimensionalen auf- oder zuklappenden Achskorrekturen sollte die Gegenkortikalis geschont und nicht völlig durchtrennt werden. In diesen Fällen empfiehlt sich eine gezielte Schwächung der Gegenkortikalis durch mehrfache Bohrungen von etwa 2,5 mm Durchmesser. Translationen und Torsionskorrekturen erfordern immer eine vollständige Knochendurchtrennung über den gesamten Querschnitt hinweg.
Die mittlere Höhe des additiven/subtraktiven Keils erlaubt Rückschlüsse auf die zu erzielende Verlängerung bzw. Verkürzung durch die Osteotomie.

Dom- und Pendelosteotomien. Sie erlauben längenneutrale Achskorrekturen in einer Ebene. Domosteotomien ( $\bullet$ Abb. 1c, d) werden bevorzugt mit einem Bohrer von 3,2-4,5 mm Durchmesser durchgeführt, am besten unter Verwendung einer Bohrlehre, die um einen Drehpunkt frei gelagert werden kann. Als Drehpunkt am Knochen eignet sich eine kräftige SchanzSchraube von $5 \mathrm{~mm}$ Durchmesser (Cave: Liegt der Drehpunkt nicht im Apex der Deformität, resultieren Translationen!).

Pendelosteotomien erfolgen mit der oszillierenden Säge. Der resultierende orts- ständige kortikospongiöse Halbschaftkeil wird von der sich öffnenden Gegenseite umgekehrt wieder eingesetzt.

Scheibenwischerosteotomien. Sie sind letztlich uniplanare Osteotomien. Bei ausgeklügelter Planung lassen sich damit sogar mehrdimensionale Deformitäten korrigieren. In ihrer „einfachen“ Form wird das distale Fragment um ein Drehzentrum scheibenwischerartig gedreht und somit die Achsausrichtung verändert. Zusätzlich sind einzeitige Verlängerungen bis zu $2 \mathrm{~cm}$ am Oberschenkel möglich.

Treppenförmige Osteotomien. Am Femur (nicht an der Tibia: Gefahr von Peronäusparese und Kompartmentsyndrom!) subtrochanter oder suprakondylär eignen sie sich 
für einzeitige Verlängerungen bis zu $3 \mathrm{~cm}$. Die entstehenden Halbschaftdefekte werden durch auto- oder allogene kortikospongiöse Knochenblöcke passgenau aufgefüllt. Für die Osteosynthese bieten sich lange $95^{\circ}$-Kondylenplatten an. Zusätzliche Achskorrekturen bis zu $5^{\circ}$ und Torsionskorrektu-

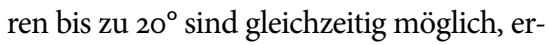
fordern allerdings eine sehr subtile Planung und exakte operative Umsetzung.

\section{Techniken der Knochendurchtrennung}

Die Wahl der geeigneten Osteotomietechnik wird durch allgemeine und lokale biologische Gegebenheiten ebenso beeinflusst wie durch die Art der geplanten Korrektur und der nachfolgenden Osteosynthese. Vorrangiges Bestreben ist immer die Schonung des ortsständigen Gewebes und insbesondere des Knochens - wesentliche Grundvoraussetzungen für eine ungestörte Knochenheilung. Dieser „biologischen Grundhaltung" steht der Wunsch nach maximaler Präzision bei der operativen Umsetzung einer Korrekturplanung allerdings häufig entgegen. In jedem Einzelfall ist daher der bestmögliche Kompromiss zwischen Biologie und mechanischer Präzision abzuwägen. Unabhängig davon, erfordern beide Zielvorgaben - Biologie und mechanische Präzision - immer gepflegte und scharfe Schneidwerkzeuge. Dies gilt für Sägeblätter, Bohrer und Meißel gleichermaßen.

\section{Oszillierende Säge}

Vorteile

- Durch glatte Schnittflächen wird ein guter Flächenkontakt erreicht.

- Sie ist geeignet für einzeitige Korrekturosteotomien mit nachfolgender Stabilisierung durch Plattenosteosynthese über denselben operativen Zugang, also für Keil- und Pendelosteotomien, uniplanare oder treppenförmige Osteotomien.

Nachteile

- Durch den relativ ausgedehnten operativen Zugang kommt es zur Weichteiltraumatisierung.

- Die knöchernen Schnittflächen werden durch Wärmeentwicklung denaturiert. Daher sollten scharfe, am besten dachverzahnte Sägeblätter verwendet und während des Sägevorgangs durch Spülung konsequent gekühlt werden.

Markraumsäge. Ihr Einsatz ist insgesamt selten indiziert.

\section{Vorteile}

- Es handelt sich um ein weichteilschonendes Verfahren zur diaphysären Durchtrennung insbesondere des Femurs und nachfolgender Marknagelosteosynthese.

- Das Verfahren ist für einzeitige Torsionskorrekturen und/oder kontinuierliche Längenkorrekturen geeignet.

Nachteile

- Für Torsionskorrekturen muss der Schnitt der Innensäge im rechten Winkel zur anatomischen Achse laufen.

- Unvollständige Schnitte sind relativ häufig und hinterlassen störende Grate, was insbesondere an der Tibia ein Nacharbeiten von außen mit dem Meißel erfordert.

- Diaphysäre Achskorrekturen frontal/ sagittal sind nur mit ergiebiger Spongiosaplastik durch Markraumbohrung (Biologie?) vertretbar.

\section{Gigli-Säge}

Vorteile

- Es handelt sich um ein weichteilschonendes Verfahren zur diaphysären Durchtrennung, insbesondere der Tibia.

- Der Einsatz ist für einzeitige Torsionskorrekturen und/oder kontinuierliche Längenkorrekturen möglich.

Nachteile

- Der subperiostale Durchzug des Gigli-Drahts ist gelegentlich technisch schwierig.

- Der Sägedraht kann klemmen und reißen. Daher empfehlen sich sanftes und gleichmäßiges Sägen ohne Unterbrechung des Sägevorgangs.

\section{Bohrer}

\section{Vorteile}

- Es handelt sich um ein weichteilschonendes Verfahren zur meta- und diaphysären Knochendurchtrennung.
Trauma Berufskrankh $2006 \cdot 8$ [Suppl 3]:

S317-S324

DOI 10.1007/s10039-005-1058-y

c) Springer Medizin Verlag 2005

W. Strecker · P. Keppler

\section{Therapie posttraumatischer Deformitäten}

\section{Zusammenfassung}

Voraussetzung für jede Korrektur von Deformitäten der oberen oder unteren Extremitäten ist eine umfassende Analyse der knöchernen Geometrie, der Gelenkfunktion und -stabilität sowie der jeweiligen Weichteilsituation. Die Planung der Deformitätenkorrektur schließt bereits die Entscheidung zu Art und Technik der Knochendurchtrennung und der nachfolgenden knöchernen Rekonstruktion ein. Die entsprechenden Kenntnisse hierzu werden im Folgenden vorausgesetzt.

\section{Schlüsselwörter}

Deformität · Knöcherne Geometrie ·

Weichteilsituation $\cdot$ Osteotomie $\cdot$ Korrektur

\section{Treatment of post-traumatic deformities}

\section{Abstract \\ Complete analysis of bony geometry, joint function and stability, and soft-tissue condi- tion is necessary before correcting deformi- ties of the upper and lower limbs. Detailed planning is mandatory and includes surgi- cal steps, type of osteotomy and bony recon- struction. Surgical procedures are presented as well as acute and continuing techniques for deformity correction.}

\section{Keywords}

Deformity · Bone geometry .

Soft-tissue condition - Osteotomy .

Correction 


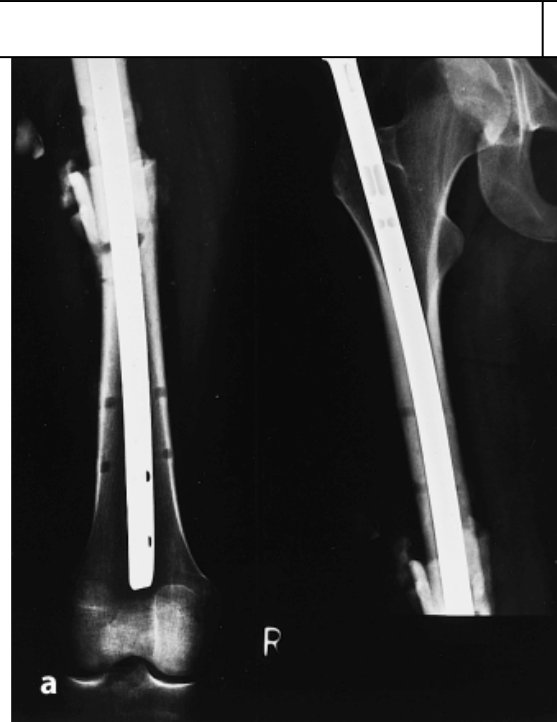

\section{Posttraumatische Deformitäten}
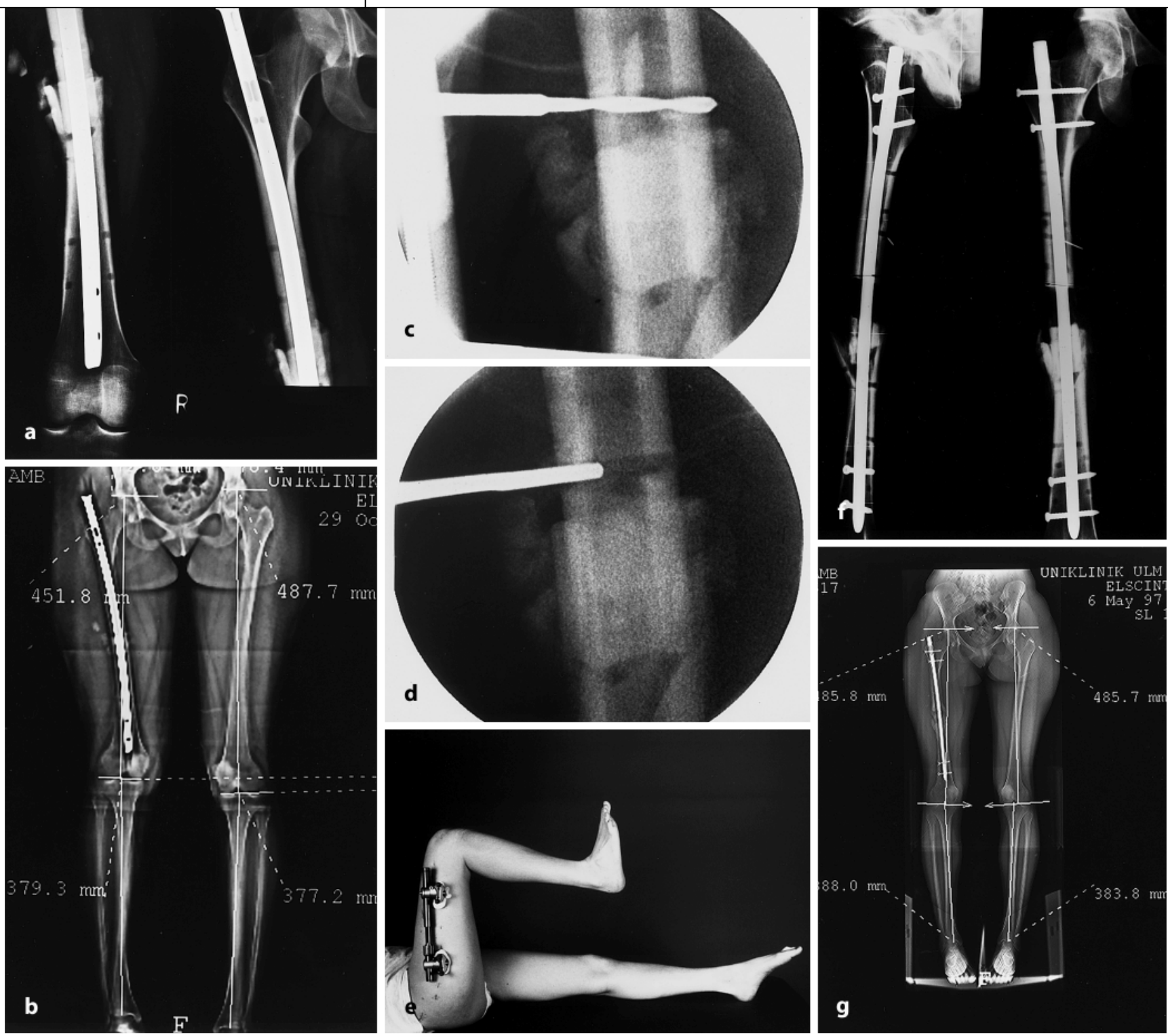

Abb. $2 \triangle$ Patientin F.N., Fall 2, a, b Verkürzung durch Teleskopage im Frakturbereich um 3,6 cm, c, d Bohrloch- und Mei-

Belosteoklasie, $\mathrm{e}$, fakute Torsionskorrektur und kontinuierliche Verlängerung durch Kallusdistraktion mittels unilatera-

lem Fixateur externe über soliden Marknagel, g Bein nach Längengewinn

- Der Bohrer ist für alle externen und distrahierenden Korrekturverfahren geeignet (• Abb. 2c).

- Es handelt sich um die für die Domosteotomie einzig mögliche Technik.

Nachteile

- Wegen fehlender glatter Schnittflächen ist das Verfahren nicht für einzeitige Torsionskorrekturen geeignet.

- Das tangentiales Bohren der Kortikalis gestaltet sich häufig schwierig, selbst mit kanülierten Bohrern, hier empfiehlt sich eine Komplettierung der Kortikalisdurchtrennung mit dem
Meißel, z. B. dem so genannten „Fähnchenmeißel“.

\section{Meißel}

Vorteile

- Es handelt sich um ein einfaches, weichteilschonendes Verfahren.

- Das Verfahren ist für alle externen und distrahierenden Korrekturverfahren, ggf. in Kombination mit dem Bohrer, geeignet (• Abb. 2d).

Nachteile

- Das Verfahren erfordert viel Geduld und handwerkliches Feingefühl.
- Es besteht die Gefahr der unkontrollierten Bruch- oder Fissurbildung. Dadurch ausgelockerte Schanz-Schrauben und Ilisarov-Drähte müssen ggf. umgesetzt werden.

\section{Korrekturtechniken}

Grundsätzlich gibt es 2 unterschiedliche Prinzipien der Deformitätenkorrektur:

- Einzeitige oder Akutkorrekturen

- Kontinuierliche oder distrahierende Verfahren 


\section{Akutkorrekturen}

Sie sind immer dann angezeigt, wenn Risiken von Dehnungsschäden und Kinking ausgeschlossen sind.

Unterschenkel. Jede akute Verlängerung, jede proximale Innentorsionskorrektur und jede proximale Varisierung bedrohen den N. peroneus und das Tibialis-anteriorKompartment. Peronäusparese und Kompartmentsyndrom sind typische und gefürchtete Komplikationen! Somit darf am Unterschenkel keine Akutverlängerung erfolgen!

Vor jeder proximalen Innentorsionskorrektur sowie Varisierung muss eine Neurolyse des $\mathrm{N}$. peroneus vorgenommen werden! Im Zweifelsfall ist eine kontinuierliche Korrektur zu indizieren!

Oberschenkel. Hier sind akute Verlängerungen proximal bis $\mathrm{zu} 3 \mathrm{~cm}$ und distal bis zu $2 \mathrm{~cm}$ möglich, ohne wesentliche Gefahr einer Überdehnung des N. ischiadicus. Dies gilt jedoch nur für einen narbenfreien Verlauf des Nervs!

Bei akuten Verkürzungen ist das Risiko eines Kinking von Blutgefäßen und Nerven abzuschätzen. Im Zweifelsfall sind kontinuierliche Verkürzungstechniken vorzuziehen.

\section{Kontinuierliche Verfahren der Kallusdistraktion}

Sie empfehlen sich bei allen Korrekturen mit potenziellen Dehnungsschäden, insbesondere bei allen Verlängerungen des Unterschenkels und bei längerstreckigen Verlängerungen des Oberschenkels (• Abb. 2).

\section{Vor- und Nachteile der Verfahren/ Verfahrenswahl}

Unabhängig von diesen Korrekturprinzipien ist die Entscheidung für eines der 3 Osteosyntheseverfahren, sei es intern, extern oder kombiniert. Generell werden Akutkorrekturen meist intern stabilisiert, kontinuierliche Korrekturen meist extern oder kombiniert intern/extern.

Vor- und Nachteile der 3 Osteosyntheseverfahren werden im Folgenden dargestellt:
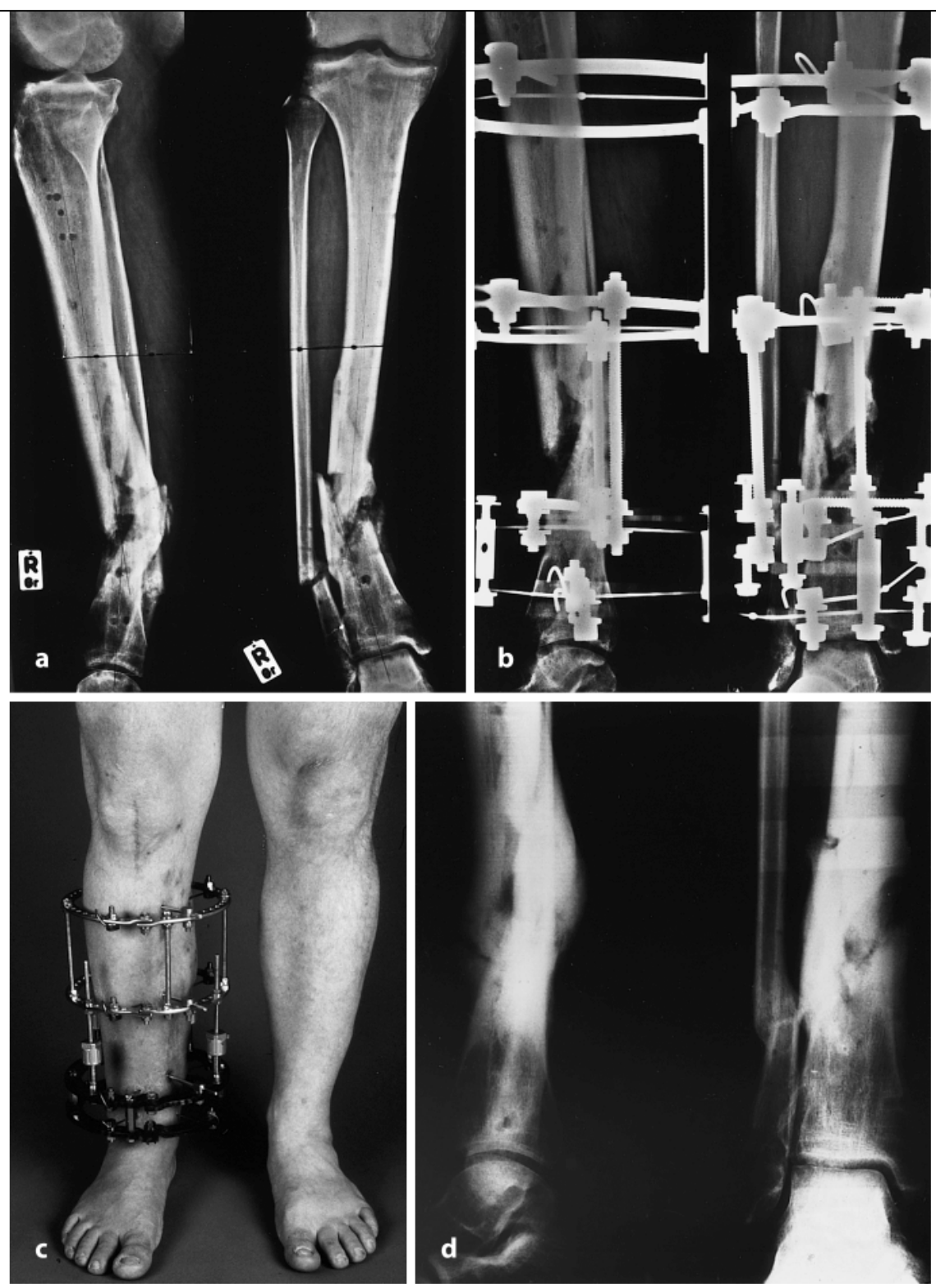

Abb. 3 \ Patient K.B., Fall 3, a Infekt-Defekt-Pseudarthrose rechter distaler Unterschenkel mit Varusdeformität, $\mathrm{b}$ kontinuierliche Achsausgradung mit gleichzeitiger Kompression der Pseudarthrose mittels Ringfixateur nach Ilisarov, $c$, d knöcherne Ausheilung und Belastungsstabilität

Interne Korrekturverfahren. Sie bieten sich bei unkritischen Weichteil- und Knochenverhältnissen an (• Abb. 1). Vorteilhaft sind ein im Allgemeinen guter Patientenkomfort mit der Möglichkeit einer frühfunktionellen Nachbehandlung. Das Infektrisiko entspricht demjenigen interner Osteosynthesen bei geschlossenen Frakturen, die gesamte Behandlungsdauer ist relativ kurz.

Nachteilig sind ein meist größeres Operationstrauma bei dem initialen Korrektureingriff und der späteren Metallentfernung. Nachkorrekturen sind nicht möglich. Operationsplanung und -technik müs- sen daher besonders präzise sein. Korrekturen mittels Marknagel können bei ausgeprägter Markraumsklerose schwierig sein oder scheitern.

Postinfektiöse Weichteil- und Knochenabschnitte sind Kontraindikationen für interne Verfahren und stellen eine Domäne externer Fixationssysteme dar. Kindliche Deformitäten werden ebenfalls bevorzugt extern korrigiert.

Kombinierte Korrekturverfahren. Sie vereinigen bei Kallusdistraktionen die Vorteile von Marknagel und externen Fixationssystemen (• Abb. 2). Während der Mark- 


\section{Posttraumatische Deformitäten}
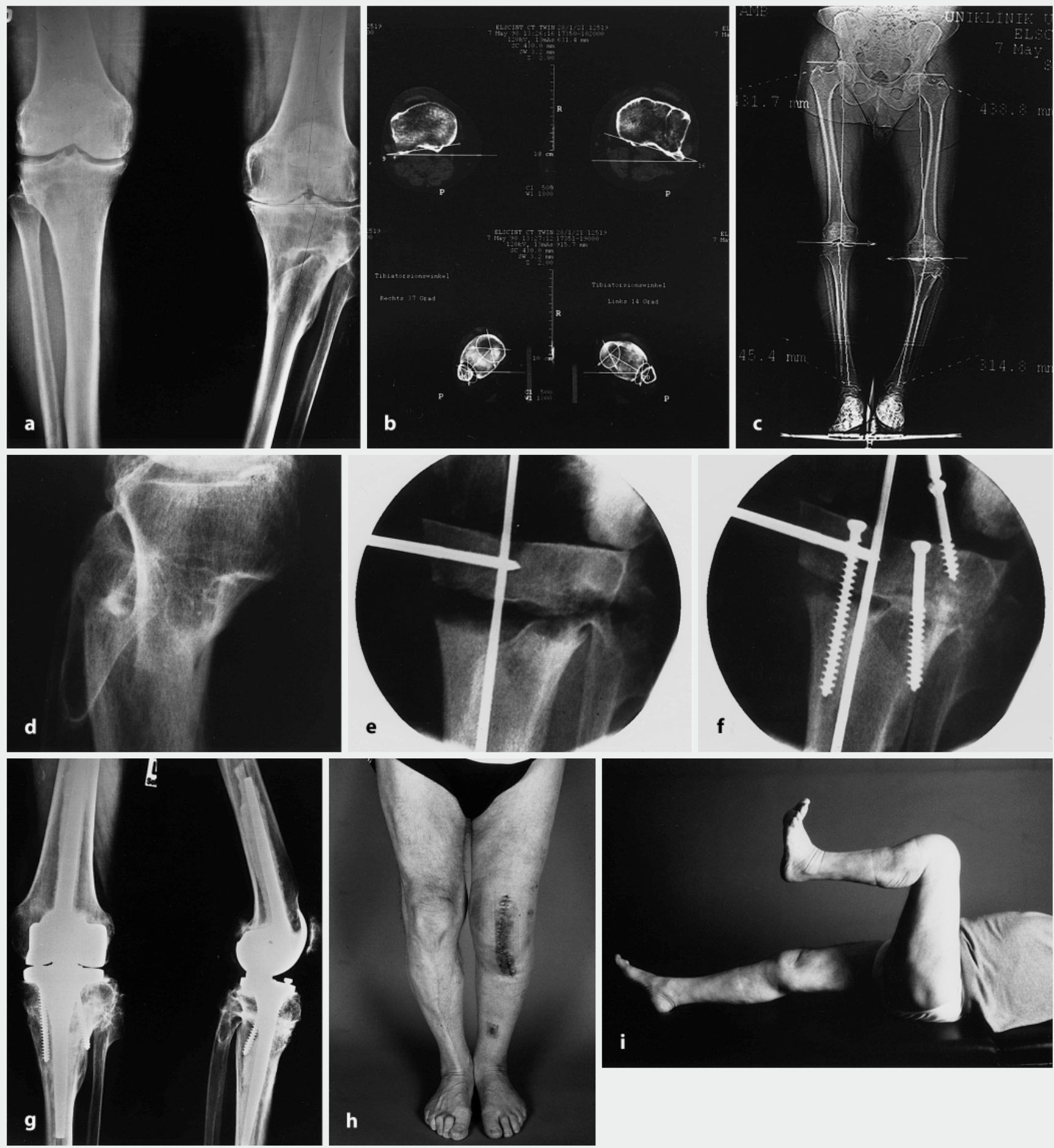

Abb. $4 \Delta$ Patient E.K., Fall 4, a-d Pangonarthrose IV, Komplexdeformität der proximalen Tibia, e, finfratuberositäre Außentorsions-/Valgisations-/Translationsosteotomie, g Knie-TEP-Implantation, $\mathrm{h}$ befriedigende Achsausgradung, i gute Kniefunktion

nagel als zentrale Leitschiene die axiale Ausrichtung sicherstellt, wird die Länge kontinuierlich durch Kallusdistraktion gewonnen. Hierbei finden bevorzugt unilaterale Distraktionsfixateure Anwendung, aber auch Ringmontagen, Hybridfixateure, Drahtseilzugsysteme und ande- res mehr. Gegenüber rein externen Systemen ist die deutlich kürzere Tragzeit des Fixateur externe bei kombinierten Verfahren ein großer Vorteil: Nach Abschluss der Kallusdistraktion werden der Marknagel statisch verriegelt und der Fixateur externe demontiert. Nachteilig ist die Gefahr einer Markraumphlegmone in einer Größenordnung von 2-5\%, begünstigt durch Pin-Track-Infektionen.

Externe Korrekturverfahren. Sie bieten bei größter Variabilität den weitesten Anwendungsbereich (•Abb. 3). Unter den 
zahlreichen Konstruktionen haben der Ringfixateur nach Ilisarov [2] und unilaterale Systeme, wie etwa der Heidelberger Fixateur [4], die weiteste Verbreitung in Mitteleuropa gefunden. Externe Fixationssysteme werden zwar auch bei Akutkorrekturen eingesetzt, ihre Domäne sind aber eigentlich jegliche Form der Kallusdistraktion, bevorzugt bei Extremitätenverlängerungen. Generell wird bei erwachsenen Nichtrauchern ein Längengewinn von durchschnittlich $1 \mathrm{~mm} /$ Tag erreicht. Die Knochenreifung und -festigung benötigen pro mm Längengewinn weitere $2 \mathrm{Ta}$ ge. Für $1 \mathrm{~mm}$ Längengewinn ist also eine durchschnittliche Tragezeit des Fixateurs von 3 Tagen anzusetzen, für $1 \mathrm{~cm}$ Längengewinn etwa 1 Monat. Hinzu kommen initial 10 Tage Ruhephase nach der Knochendurchtrennung vor Beginn der Kallusdistraktion. Bei Rauchern sind die genannten Zeiten häufig wesentlich länger, Störungen der Kallusreifung kommen ebenfalls öfter vor.

Die relativ langen Tragezeiten der externen Fixationen und die gelegentlich ausladenden Konstruktionen machen gleichzeitig deren Nachteile verständlich:

- Geringer Patientenkomfort

- Hohe Rate an Pin-Track-Infektionen (bis zu 95\%)

- Erschwerte Rehabilitation durch pinbedingte Transfixierung von Weichteilen

Während die Vorteile der externen Fixationen unbestritten sind, insbesondere bei delikaten Weichteil- und Knochenverhältnissen, erfordern die genannten Nachteile eine adäquate Patientencompliance und eine besonders belastungsfähige Kooperation zwischen Arzt und Patient. Nur so kann den entsprechenden Komplikationen rechtzeitig und angemessen begegnet werden. Pin-Track-Infektionen sind so schnell als möglich durch systemische Antibiose zu behandeln. Gelegentlich werden lokale Weichteilrevisionen oder ein Umsetzen der Pins nötig.

\section{Achskorrekturen mit gleichzeitigem Gelenkersatz}

In seltenen Fällen sind Beindeformitäten mit höhergradigen Panarthrosen angren- zender Gelenke vergesellschaftet. Bei derartigen Konstellationen ist durch alleinige Achskorrekturen keine Besserung der Gelenksymptomatik zu erwarten. Andererseits ändert ein isolierter Gelenkersatz nichts an der knöchernen Deformität und der dadurch induzierten Fehlbelastung benachbarter Gelenke. In diesen insgesamt seltenen Situationen kann sich daher eine Achskorrektur mit oder durch gleichzeitigen Gelenkersatz als vorteilhaft erweisen (• Abb. 4).

\section{Kasuistiken}

\section{Fall 1}

Bei der 41-jährigen Patientin R.E. resultierten 15 Jahre nach bilateralen Unterschenkelfrakturen und Oberschenkelfraktur links eine Deformität des linken suprakondylären Oberschenkels von $12^{\circ}$ Varusund $9^{\circ}$ Außentorsionsabweichung sowie eine Innentorsionsdeformität des linken Unterschenkels von $26^{\circ}$. Darüber hinaus lag eine medial betonte Pangonarthrose vor (• Abb. 1a, b).

Nach arthroskopischer Bestätigung und Revision des Kniebinnenbefunds wurde zunächst eine suprakondyläre Domosteotomie (• Abb. 1c) mit $14^{\circ}$ Valgisation und $10^{\circ}$ Innentorsion über retrograden Marknagel vorgenommen. SchanzSchrauben von $5 \mathrm{~mm}$ Durchmesser zeigten das Ausmaß dieser Korrektur in der Frontalebene und bezüglich der Torsionsverhältnisse an (• Abb. 1d, e). Nach Abschluss der suprakondylären Femurkorrektur erfolgte die diaphysäre Außentorsionskorrektur der Tibia um $25^{\circ} \mathrm{mit}$ tels Gigli-Osteotomie (• Abb. 1f, g) unter Nutzung von Schanz-Schrauben proximal und distal der Osteotomie als Zeiger für das Ausmaß der Torsionskorrektur (• Abb. 1h, i).

Die Torsionssicherung erfolgt bei torsionsstabilen Marknägeln durch statische Verriegelung, andernfalls durch zusätzliche Plattenosteosynthese.

\section{Fall 2}

Bei der 17-jährigen Patientin F.N. bestanden nach unverriegelter Marknagelosteosynthese einer diaphysären Femurfraktur rechts eine Verkürzung durch Teleskopage im Frakturbereich um 3,6 cm (• Abb. 2a, b) und eine Innentorsionsabweichung von $16^{\circ}$. Es wurden eine Bohrloch- und Meißelosteoklasie (• Abb. 2c, d) mit akuter Torsionskorrektur und kontinuierlicher Verlängerung durch Kallusdistraktion (• Abb. 2e) mittels unilateralem Fixateur externe über einen soliden Marknagel (• Abb. 2f) vorgenommen. Nach entsprechendem Längengewinn (• Abb. 2g) erfolgten die statische Verriegelung und Demontage des Fixateur externe.

\section{Fall 3}

Die offene Fraktur ( $\mathrm{AO} 42-\mathrm{C}_{3} .3$; $\mathrm{OII}^{\circ}$ ) des 38-jährigen Patienten K.B. war primär durch Fixateur externe stabilisiert worden. Nach Verfahrenswechsel auf soliden Marknagel und späteren Nagelbruch, Verfahrenswechsel auf gebohrten Marknagel, Spongiosaplastik u.a.m. kam es 8 Monate später zu einer Infekt-Defekt-Pseudarthrose des rechten distalen Unterschenkels mit Varusdeformität (• Abb. 3a).

Es wurde eine kontinuierliche Achsausgradung mit gleichzeitiger Kompression der Pseudarthrose mittels Ringfixateur nach Ilisarov ( $\bullet$ Abb. $3 \mathbf{b})$ vorgenommen, worunter es zur knöchernen Ausheilung und Belastungsstabilität kam (• Abb. 3c, d).

\section{Fall 4}

Bei dem Patienten E.K., 77 Jahre, resultierte 53 Jahre nach Schussbruch des linken Tibiakopfs mit konsekutiver Osteitis neben einer wackelsteifen Pangonarthrose IV mit einem Bewegungsumfang von o$30-55^{\circ}$ eine Komplexdeformität der proximalen Tibia mit $14^{\circ}$ Varus- und $29^{\circ}$ Innentorsionsabweichung, $2,3 \mathrm{~cm}$ Verkürzung und 2,0 cm posteriorer Translation des Tibiaschafts (• Abb. 4a-d).

Nach infratuberositärer Außentorsions- $\left(20^{\circ}\right) /$ Valgisations- $\left(15^{\circ}\right) /$ Translationsosteotomie $(2,0 \mathrm{~cm})$ autogen additiv (- Abb. 4e, f) wurde eine gekoppelte KnieTEP (• Abb. 4g) implantiert. Es wurde eine befriedigende Achsausgradung frontal (- Abb. 4h) und sagittal mit guter Kniefunktion (• Abb. 4i) erreicht. 


\section{Korrespondierender Autor}

\section{Prof. Dr. W. Strecker}

Klinik für Orthopädische Chirurgie

und Unfallchirurgie, Klinikum Bamberg,

Buger Straße 80, 96049 Bamberg

E-Mail: chirurgie2@sozialstiftung-bamberg.de

Interessenkonflikt: Es besteht kein Interessenkonflikt. Der korrespondierende Autor versichert, dass keine Verbindungen mit einer Firma, deren Produkt in dem Artikel genannt ist, oder einer Firma, die ein Konkurrenzprodukt vertreibt, bestehen. Die Präsentation des Themas ist unabhängig und die Darstellung der Inhalte produktneutral.

\section{Literatur}

1. Baumgart R, Kettler M, Zeiler C et al. (1998) Indikation und Technik der Knochendurchtrennung. Chirurg 69: 1188-1196

2. Ilisarov GA (1992) Transosseus synthesis. Springer, Berlin Heidelberg New York

3. Pfeil J, Grill F, Graf R (1996) Extremitätenverlängerung, Deformitätenkorrektur, Pseudarthrosenbehandlung. Springer, Berlin Heidelberg New York

4. Strecker W, Keppler P (2002) Analyse und Korrektur von Beindeformitäten. Teil 1: Analyse. Unfallchirurg 73: 811-829 